\title{
Estimates for the Roots of Polynomials Without Complex Analysis
}

\author{
Ismaïla Diouf ${ }^{1}$ \\ ${ }^{1}$ Deptartment of Maths-Info FST, Université Cheikh Anta DIOP, Dakar, Senegal \\ Correspondence: Ismaïla Diouf, Deptartment of Maths-Info FST, Université Cheikh Anta DIOP, Dakar, Senegal. \\ E-mail: ismaila.diouf@ucad.edu.sn
}

Received: November 11, 2013 Accepted: December 15, 2013 Online Published: December 30, 2013

doi:10.5539/jmr.v6n1p42

URL: http://dx.doi.org/10.5539/jmr.v6n1p42

\begin{abstract}
In our precedence paper (Diouf, Diakhate, \& Watt, 2013), we show the continuity of the zeros of a univariate polynomial which respect to the coefficients. Here we study the sizes of a polynomial and their bounds. The main originality of this paper is maybe a definition of the measure of a polynomial without any reference to the roots, this leads to a very elementary proof of bounds for the factors of polynomials, a subject which is also revisited here. Most of our proofs are extremely simple and all are quite elementary.
\end{abstract}

Keywords: polynomials, roots, size of a polynomial, separation of roots, measure of a polynomial

\section{Bounds for the Roots}

\subsection{Bounds for All the Roots}

We consider a field $K$ equipped by a non-trivial absolute value, i.e a map $x \mapsto|x|$ from $K$ to $\mathbb{R}$ such that

$$
\forall x,|x| \geqslant 0, \quad|x|=0 \Rightarrow x=0, \quad \forall x, y,|x+y| \leqslant|x|+|y|, \quad|x y|=|x||y| .
$$

If there exists $x \in K^{*}$ with $|x| \neq 1$, then clearly the set of absolute values of elements of $K$ contains arbitrary large and arbitrary non zero values.

We also need a lemma to bound for the roots of polynomials, this result is essentially due to Cauchy (1891).

Lemma 1 Let $P(X)=X^{d}+a_{d-1} X^{d-1}+\cdots+a_{0}$ be a polynomial with coefficients in $K$. If a positive real number $r$ satisfies the condition

$$
r^{d} \geqslant\left|a_{d-1}\right| r^{d-1}+\cdots+\left|a_{0}\right|
$$

and if $z \in K$ is a root of $P$ then $|z| \leqslant r$.

Proof. For the proof of this lemma, see Diouf (2007).

This lemma enables us to give many inequalities for the complex roots of a polynomial, most of which are due to Cauchy (1891). Here are two of them.

Theorem 1 Let $P(X)=X^{d}+a_{1} X^{d-1}+\cdots+a_{d}$ be a monic polynomial with coefficients in $K$ and let $z \in K$ be a root of $P$. Then $z$ satisfies the following inequalities:

i) $|z|<1+\max \left|a_{k}\right| ; 1 \leqslant k \leqslant d$. If the coefficients of $P$ are equal to zero for $j \notin\left\{j_{1}, j_{2}, \ldots, j_{k}\right\}$ and if $\lambda_{j_{1}}, \ldots, \lambda_{j_{k}}$ are positive real numbers which satisfy

$$
\lambda_{j_{1}}^{-1}+\lambda_{j_{2}}^{-1}+\cdots+\lambda_{j_{k}}^{-1} \leqslant 1
$$

then

ii) $|z| \leqslant \max _{1 \leqslant h \leqslant k}\left\{\left(\lambda_{j_{h}}\left|a_{j_{h}}\right|\right)^{1 / j_{h}}\right\}$.

Proof. Let us prove the upper bound i).

Let $A$ be the maximum of the quantities $\left|a_{k}\right|$ for $1 \leqslant k \leqslant d$. Without loss of generallity, we may suppose that $a_{d}$ is nonzero, thus $A>0$. Let $f$ be the function defined in the proof of Lemma 1 . Then, 


$$
\begin{aligned}
f(1+A) & =(1+A)^{d}-\left|a_{1}\right|(1+A)^{d-1}-\cdots-\left|a_{d-1}\right|(1+A)-\left|a_{d}\right| \\
& \geqslant(1+A)^{d}-A(1+A)^{d-1}-\cdots A(1+A)-A \\
& =(1+A)^{d}-A \frac{(1+A)^{d}-1}{1+A-1} \\
& =1 .
\end{aligned}
$$

Hence the result.

Consider now the property ii).

Let us call $r$ the right hand-side of inequality ii). Then, by definition, the inequalities

$$
\left|a_{j_{h}}\right| \leqslant \lambda_{j_{h}}^{-1} r^{j_{h}}
$$

hold for $1 \leqslant h \leqslant k$.

Hence the upper bound

$$
\sum_{h=1}^{k}\left|a_{j_{h}}\right| r^{d-j_{h}} \leqslant \sum_{h=1}^{k} r^{d} \lambda_{j_{h}}^{-1} \leqslant r^{d} .
$$

Which can also be written as $f(r) \geqslant 0$. Hence the result, using again Lemma 1.

Corollary 1 Let $P(X)=a_{0} X^{d}+a_{1} X^{d-1}+\cdots+a_{d}$ be a polynomial in $K[X]$ with $a_{d} \neq 0$. Then every root $z \in K$ of $P$ satisfies the inequality

$$
|z|>\left(1+\max \left\{\left|\frac{a_{0}}{a_{d}}\right|,\left|\frac{a_{1}}{a_{d}}\right|, \ldots,\left|\frac{a_{d-1}}{a_{d}}\right|\right\}\right)^{-1} .
$$

Proof. Consider the polynomial $P^{*}=X^{d} P\left(X^{-1}\right)$, called the reciprocal polynomial of $P$. Then the conclusion follows directly from i) applied to $P^{*}$, polynomial whose roots are the inverses of the roots of $P$.

Remark Of course, the argument used in the proof of the Corollary shows that every upper bound of the modules of the roots of $P^{*}$ leads to a lower bound of those of $P$ when $P(0) \neq 0$. We leave to the reader the similar application of inequality ii).

Corollary 2 Let $P(X)=X^{d}+a_{1} X^{d-1}+\cdots+a_{d}=\left(X-\alpha_{1}\right) \cdots\left(X-\alpha_{d}\right) \in K[X]$ be a monic polynomial which splits completely in $K$ and put

$$
K(P)=\max \left\{\left|a_{j}\right|^{1 / j} ; 1 \leqslant j \leqslant d\right\} \quad \text { and } \rho(P)=\max \left\{\left|\alpha_{j}\right| ; 1 \leqslant j \leqslant d\right\} .
$$

Then

$$
\rho(P) \leqslant 2 K(P) \quad \text { and } \quad K(P) \leqslant d \rho(P) .
$$

Proof. The first inequality is a special case of inequality ii) of Theorem 1 (take $\lambda_{j}=2^{-j}$ for $j=1,2, \ldots, d$ ). The second inequality is an easy consequence of Vieta's formula. Indeed,

$$
\left|a_{j}\right|=\left|\sum_{1 \leqslant i_{1}<\cdots<i_{j} \leqslant d} \alpha_{i_{1}} \cdots \alpha_{i_{j}}\right| \leqslant\left(\begin{array}{l}
d \\
j
\end{array}\right) \rho(P)^{j},
$$

and the result follows from the trivial inequality

$$
\left(\begin{array}{l}
d \\
j
\end{array}\right) \leqslant d^{j}
$$

\subsection{Bounds for Some Roots}

In this section, we indicate two results dealing with several roots of a polynomial. 
Proposition 1 Let $P=a_{d} X^{d}+a_{d-1} X^{d-1}+\cdots+a_{0} \in K[X]$ be a polynomial. Suppose that $\alpha_{1}, \ldots, \alpha_{k} \in K$ are some roots of $P$ and put

$$
P=\left(X-\alpha_{1}\right) \cdots\left(X-\alpha_{k}\right) Q \quad \text { and } \quad H(P)=\max _{0 \leqslant j<d}\left|a_{j}\right| .
$$

Then

$$
\left\|\alpha_{1}-1|\cdots| \alpha_{k}-1\right\| \cdot H(Q)<H(P),
$$

where $H(Q)$ is the maximum of the absolute values of the coefficients of $Q$.

Proof. This result has been proved in Mignotte (1991). The proof is very simple. First, notice that this is enough to prove this inequality for $k=1$, then the general case follows by induction. Assume that

$$
P=(X-\alpha) Q, \quad \text { where } \quad Q=b_{d} X^{d}+b_{d-1} X^{d-1}+\cdots+b_{0} .
$$

Suppose first that $|\alpha| \geqslant 1$ and let $i$ be the minimal index such that $H(Q)=\left|b_{i}\right|$. The relation

$$
a_{i}=b_{i-1}-\alpha b_{i}, \quad \text { where } \quad b_{d}=b_{-1}=0,
$$

implies

$$
H(P) \geqslant\left|a_{i}\right| \geqslant|\alpha| H(Q)-\left|b_{i-1}\right|>(|\alpha|-1) H(Q),
$$

and the result follows.

When $|\alpha|<1$ the proof is similar (consider now the greatest index $j$ such that $H(Q)=\left|b_{j}\right|$ ), we omit the details.

Using the notion of compound matrices Specht (1938) proved the following result, for which we do not reproduce the proof. We just notice that the original proof was given for complex numbers but that it works in the case of a field $K$ with an absolute value.

Proposition 2 Let $P=a_{d} X^{d}+a_{d-1} X^{d-1}+\cdots+a_{0} \in K[X]$ be a monic polynomial. Suppose that $\alpha_{1}, \ldots, \alpha_{k} \in K$ are some roots of $P$. Then

$$
\left|\alpha_{1} \cdots \alpha_{k}\right| \leqslant k H(P)+1, \quad \text { where } H(P)=\max _{0 \leqslant j<d}\left|a_{j}\right| .
$$

We also notice that the proof uses Gerschghorin bounds for the eigenvalues of a matrix and that these bounds still hold for matrices with coefficients in $K$ (the proof being the same).

\section{Different Notions of "Size" for Polynomials}

As in the previous section, $K$ is a field equipped with an absolute value. We consider a polynomial

$$
P(X)=a_{0} X^{d}+a_{1} X^{d-1}+\cdots+a_{d} \in K[X] .
$$

We define the (naïve) height of $P$ by

$$
H(P)=\max \left|a_{j}\right| ; 0 \leqslant j \leqslant d,
$$

as in Proposition 1 and 2 above. Then

$$
H(P \cdot Q) \leqslant(d+1) H(P) \cdot H(Q), \quad \text { where } \quad d=\min \{\operatorname{deg} P, \operatorname{deg} Q\} .
$$

We define the length of $P$ by

$$
L(P)=\sum_{j=0}^{d}\left|a_{j}\right| .
$$

Then

$$
L(P \cdot Q) \leqslant L(P) \cdot L(Q)
$$

We define the norm of $P$ by

$$
N(P)=\|P\|_{\infty}=\sup \{|P(z)| ;|z|=1\}
$$

Then

$$
N(P \cdot Q) \leqslant N(P) \cdot N(Q) .
$$

The following result is trivial but neverthless it is usefull. 
Lemma 2 Let $P, Q$ qnd $R$ be polynomials in $K[X]$, with $P=Q R$. Then

$$
\inf \{|Q(z)| ;|z|=1\} \cdot N(R) \leqslant N(P) .
$$

Moreover, if $Q=\left(X-\alpha_{1}\right) \cdots\left(X-\alpha_{k}\right)$ splits in $K$, then

$$
|| \alpha_{1}|-1| \cdots|| \alpha_{k}|-1| \cdot N(R) \leqslant N(P) .
$$

These different sizes satisfy the following obvious inequalities for any $P \in K[X]$,

$$
H(P) \leqslant L(P) \leqslant(1+\operatorname{deg} P) H(P), \quad N(P) \leqslant L(P) .
$$

Moreover, if $K$ contains primitive roots of unity of arbitrary large order $n$ coprime to the characteristic of $K$ then let $n>d$ be coprime with the characteristic of $K$ and let $\zeta$ be a primitive $n$-th root of unity. Then it is easy to verify that if

$$
P(X)=\sum_{k=0}^{d} a_{k} X^{k}
$$

Then, since

$$
\sum_{j=0}^{n-1} \zeta^{r_{j}}= \begin{cases}n, & \text { if } n \mid r \\ 0, & \text { otherwise }\end{cases}
$$

we get

$$
n a_{k}=\sum_{j=0}^{n-1} \zeta^{-k j} P\left(\zeta^{j}\right)
$$

dor any $k=0, \ldots, d$. Indeed,

$$
\sum_{j=0}^{n-1} \zeta^{-k j} P\left(\zeta^{j}\right)=\sum_{h=0}^{d} a h \sum_{j=0}^{n-1} \zeta^{(h-k) j}
$$

and the result follows from (5).

For example, in the case $K=\mathbb{C}$ this implies

$$
H(P) \leqslant N(P) .
$$

We can obtain a similar, but weaker result, with more general hypotheses. Suppose that $K$ contains more than $d$ different elements of absolute value equal to 1 and consider a nonzero polynomial $P \in K[X]$ of degree $d$. Chose $d+1$ elements $x_{0}, x_{1}, \ldots, x_{d}$ of $K$ with $\left|x_{i}\right|=1$ for all $i$. Then $P$ can be interpolated at the points $x_{0}, \ldots, x_{d}$ and its coefficients are given by Lagrange's formulas (for example). This study yields to the following result.

Proposition 3 Let $d$ be a given positive integer. Suppose that $K$ contains more than d different elements of absolute value equal to 1 and consider any nonzero polynomial $P \in K[X]$ of degree $d$. Then there exists a constant $C_{d}$ independent of $P$ such that

$$
H(P) \leqslant C_{d}\|P\|_{\infty} .
$$

Moreover, if $K$ contains primitive roots of unity of order $n>d$, with $n$ coprime to the characteristic of $K$, and let $\zeta$ be the primitive $n-t h$ root of unity. Then if $P=a_{d} X^{d}+a_{d-1} X^{d-1}+\cdots+a_{1} X+a_{0}$, the coefficients $a_{k}$ satisfies

$$
n a_{k}=\sum_{j=0}^{n-1} \zeta^{k j} P\left(\zeta^{j}\right)
$$

for any $k=0, \ldots, d$. For example, in the case $K=\mathbb{C}$ this implies

$$
H(P) \leqslant N(P)
$$

\section{The Dandelin-Graeffe Transformation}

Definition 1 According to Diouf (2007), the principles of this method are well presented. 
Let $K$ be any field (we could even work in any ring) and $P \in K[X]$. We define formally the Dandelin-Graeffe Transformation as

$$
P(X) \mapsto P(\sqrt{X}) P(-\sqrt{X}) .
$$

Decompose $P$ in its "even" part and its "odd" part, i.e.

$$
P(X)=F\left(X^{2}\right)+X G\left(X^{2}\right) .
$$

Then we have the following result.

Proposition 4 With the above notation,

$$
P_{1}(X)=P(\sqrt{X}) P(-\sqrt{X})
$$

is a polynomial with coefficients in $K$ which satisfies

$$
P_{1}(X)=F^{2}(X)-X G^{2}(X)
$$

and if $\alpha_{1}, \alpha_{2}, \ldots, \alpha_{d}$ are the roots of $P$ in a field $L$ containing $K$ then the roots of $P_{1}$ in $L$ are the squares of $\alpha_{j}$ 's.

Proof. Formally,

$$
\begin{aligned}
P_{1}(X) & =P(\sqrt{X}) P(-\sqrt{X}) \\
& =(F(X)+\sqrt{X} G(X))(F(X)-\sqrt{X} G(X)) \\
& =F^{2}(X)-X G^{2}(X),
\end{aligned}
$$

which prove the above formula and - at the same time- that $P_{1}$ is indeed a polynomial with coefficients in $K$.

The third assertion follows from the relations

$$
\begin{aligned}
P_{1}(X) & = \pm a_{d}^{2}\left(\sqrt{X}-\alpha_{1}\right) \cdots\left(\sqrt{X}-\alpha_{d}\right)\left(\sqrt{X}+\alpha_{1}\right) \cdots\left(\sqrt{X}+\alpha_{d}\right) \\
& = \pm a_{d}^{2}\left(X-\alpha_{1}^{2}\right) \cdots\left(X-\alpha_{d}^{2}\right) .
\end{aligned}
$$

For the transformation $P \mapsto P_{1}$ we also use the notation

$$
P_{1}=\mathcal{G}(P)
$$

Notice that $\mathcal{G}$ is "multiplicative":

$$
\mathcal{G}(P \cdot Q)=\mathcal{G}(P) \mathcal{G}(Q)
$$

It is easy to verify that

$$
L\left(P_{1}\right) \leqslant L^{2}(P) .
$$

The fact that the roots of $P_{1}$ are the squares of the roots of $P$ is the origin of the Dandelin-Graeffe's method.

Suppose that $P \in \mathbb{R}[X]$ is a polynomial of degree $d$ with the roots $\alpha_{1}, \ldots, \alpha_{d} \in \mathbb{C}$, and assume moreover that $P$ has a unique dominant root, let $\alpha_{1}$. Consider the sequence of transformation $P_{0}=P, P_{1}=\mathcal{G}(P), \ldots, P_{k}=\mathcal{G}\left(P_{k-1}\right.$. Then the roots of $P_{k}$ are the $\alpha_{i}^{2^{k}}$, s and $\left|\alpha_{1}\right|^{2^{k}}$ is much greater than any $\left|\alpha_{i}^{2^{k}}\right|$ for $i=2, \ldots, d$. This shows that, if we denote

$$
P_{k}(X)=a_{0}^{(k)} X^{d}+a_{1}^{(k)} X^{d-1}+\cdots
$$

then

$$
-\frac{a_{1}^{(k)}}{a_{0}^{(k)}} \simeq \alpha_{1}^{2^{k}},
$$

thus

$$
\left|\frac{a_{1}^{(k)}}{a_{0}^{(k)}}\right|^{2^{-k}} \underset{k \rightarrow \infty}{\longrightarrow}\left|\alpha_{1}\right| .
$$

We put $\rho(P)=\left|\alpha_{1}\right|$. In other words, the Dandelin-Graeffe method furnishes the value of $\rho(P)$, when $P$ has a unique dominant root. 
Now a glance shows that the previous proof holds in any field $K$ with an absolute value, provided that the roots of $P$ belong to $K$ and that there is a unique dominant root.

\subsection{A Computation of $\rho(P)$ in the General Case}

Here we consider $P \in K[X]$, a field with an absolute value and we assume that $P$ splits in $K$ and that its roots are $\alpha_{1}, \ldots, \alpha_{d}$ belong to $K$ and satisfies $\alpha_{1} \leqslant \cdots \alpha_{d}$, thus $\rho(P)=\left|\alpha_{1}\right|$.

In section 2 we have seen that

$$
\rho(P) \leqslant 2 K(P) \quad \text { and } \quad K(P) \leqslant d \rho(P),
$$

where $d$ is the degree of $P$. This implies

$$
\frac{K(P)}{d} \leqslant \rho(P) \leqslant 2 K(P)
$$

If we apply these inequalities to $P_{k}=\mathcal{G}^{k}(P)$ we get

$$
\frac{K\left(P_{k}\right)}{d} \leqslant \rho^{2^{k}}(P) \leqslant 2 K\left(P_{k}\right)
$$

which prove that

$$
K\left(P_{k}\right)^{2^{-k}} \underset{k \rightarrow \infty}{\longrightarrow} \rho(P) .
$$

In other words, the Dandelin-Graeffe's method can be used to compute $\rho(P)$ for $P \in K[X]$, even if there is no dominant root. For the case of polynomials with complex coefficients, the result was proved by Davenport-Mignotte (1990).

Remark When $\left|a_{d}\right|>0$, working with the polynomial $P^{*}$, this method gives also an approximate value of $\left|\alpha_{d}^{-1}\right|$, hence of $\left|\alpha_{d}\right|$.

\subsection{A Computation of the $\left|\alpha_{j}\right|$ 's in the General Case}

It is easy to see that in the case of a polynomial whose roots $\alpha_{1}, \ldots, \alpha_{d}$ satisfy $\left|\alpha_{1}\right|>\left|\alpha_{2}\right|>\cdots\left|\alpha_{d}\right|$ then the classical Dandelin-graeffe method permits to compute approximate values for all the $\left|\alpha_{j}\right|$ 's. Here we want to generalize the method of the previous section to make this computation without any special hypothesis on the roots.

We suppose that $P \in K[X]$ is a monic polynomial (this assumption just to have simpler formulas) whose roots $\alpha_{1}, \ldots, \alpha_{d}$ all belong to $K$ and satisfy $\left|\alpha_{1}\right| \geqslant\left|\alpha_{2}\right| \geqslant \cdots \geqslant\left|\alpha_{d}\right|$.

Suppose that for some $l$ we have $\left|\alpha_{l}\right|>\left|\alpha_{l+1}\right|$ then the $l$ th elementary symmetric function of the roots of $P$ is

$$
\sigma_{l}=\sum_{1 \leqslant i_{1}<\cdots<i_{l} \leqslant d} \alpha_{i_{1}} \cdots \alpha_{i_{l}}=\alpha_{1} \cdots \alpha_{l}+\text { "smaller terms". }
$$

This implies that

$$
\left|a_{l}^{(k)}-(-1)^{l}\left(\alpha_{1} \cdots \alpha_{l}\right)^{2^{k}}\right| \leqslant\left(\begin{array}{l}
d \\
l
\end{array}\right)\left|\alpha_{1} \cdots \alpha_{l-1} \alpha_{l+1}\right|^{2^{k}}
$$

hence

$$
\left|a_{l}^{k}\right|^{2^{-k}} \underset{k \rightarrow \infty}{\longrightarrow}\left|\alpha_{1} \cdots \alpha_{l}\right|, \quad\left|\alpha_{l}\right|>\left|\alpha_{l+1}\right|
$$

For example:

$$
\left|a_{2}^{k}\right|^{2^{-k}} \underset{k \rightarrow \infty}{\longrightarrow}\left|\alpha_{1} \cdots \alpha_{l}\right|, \quad\left|\alpha_{2}\right|>\left|\alpha_{3}\right| .
$$

One can also notice that we always have

$$
\left|a_{j}^{(k)}\right| \leqslant\left(\begin{array}{c}
d \\
j
\end{array}\right)\left|\alpha_{1} \cdots \alpha_{j}\right|^{2^{k}}
$$

which implies

$$
\forall j, \quad\left|\alpha_{1} \cdots \alpha_{j}\right| \leqslant \lim _{k \rightarrow+\infty} \sup \left|a_{j}^{(k)}\right|^{2^{-k}}
$$

One usefull question is 
Question 1 How to recognize the case $\left|\alpha_{l}\right|>\left|\alpha_{l+1}\right|$ ?

It will help to improve the efficiency of the calculations. We'll try to give a detailled analysis and a possible answer to this question in another paper.

Example 1 Suppose that the degree of the polynomial $P$ is 4 and that its roots satisfy $\left|\alpha_{1}\right| \geqslant\left|\alpha_{2}\right| \geqslant\left|\alpha_{3}\right| \geqslant\left|\alpha_{4}\right|$. By the method of the previous section section, we can compute approximate values of $\left|\alpha_{1}\right|$ and $\left|\alpha_{4}\right|$ (see previous Remark). Now there are two cases:

$\bullet$ if $\left|\alpha_{2}\right|>\left|\alpha_{3}\right|$, then

$$
\left|\alpha_{2}\right|=\frac{1}{\left|\alpha_{1}\right|} \lim _{k \rightarrow \infty}\left|a_{2}^{(k)}\right|^{2^{-k}}
$$

and

$$
\left|\alpha_{3}\right|=\frac{a_{4}}{\alpha_{1} \alpha_{2} \alpha_{4}},
$$

- if $\left|\alpha_{2}\right|=\left|\alpha_{3}\right|$, then

$$
\left|\alpha_{2}\right|=\left|\alpha_{3}\right|=\sqrt{\frac{a_{4}}{\left|\alpha_{1} \alpha_{4}\right|}}
$$

\subsection{The Measure of a Polynomial}

For $P \in \mathbb{C}[X]$ the measure was defined in 1955 by K. Mahler as follows. If

$$
P=a\left(X-\alpha_{1}\right) \cdots\left(X-\alpha_{d}\right)
$$

then

$$
M(P)=|a| \prod_{j=1}^{d} \max \left\{1,\left|\alpha_{j}\right|\right\} .
$$

In Cerlienco-Mignotte-Piras (1987), it was proved that (with the notation of the previous section)

$$
L\left(P_{k}\right)^{2^{-k}} \underset{k \rightarrow \infty}{\longrightarrow} M(P)
$$

Here, for $P \in K[X]$, we put, by the definition,

$$
M(P):=\lim _{k \rightarrow \infty} L\left(P_{k}\right)^{2^{-k}} .
$$

The fact that $\left|a_{0}\right| \leqslant L\left(P_{1}\right)^{1 / 2} \leqslant L(P),\left|a_{0}\right| \leqslant L\left(P_{2}\right)^{1 / 4} \leqslant L_{1}(P)^{1 / 2}, \cdots$ shows that the sequence $\left.L\left(P_{k}\right)^{2^{-k}}\right)$ is nonincreasing and bounded below by $\left|a_{0}\right|$, this proves that this limit exists and that

$$
\left|a_{0}\right| \leqslant M(P) \leqslant L(P) .
$$

In the case $K=\mathbb{C}$, the field of complex numbers, it is known that

$$
M(P) \leqslant\|P\|,
$$

where $\|P\|=\left(\sum_{j=0}^{d}\left|a_{j}\right|^{2}\right)^{1 / 2}$ (reference: Landau, 1905).

Question 2 Is it true in general that $M(P) \leqslant\|P\|$ ?

We know that it is true in the complex field and we think that it still be true in every field equiped with an adequate norm. It is not very important for the scope of this paper. It's just a curiosity.

Notice that we also have, using (4),

$$
H\left(P_{k}\right)^{2^{-k}} \underset{k \rightarrow \infty}{\longrightarrow} M(P)
$$

The measure satisfies also the elementary following property

$$
M\left(P\left(X^{k}\right)\right)=M(P), \quad \text { for any integer } k \geqslant 2 .
$$

From the above results, it is clear that

$$
M(P \cdot Q) \leqslant M(P) \cdot M(Q)
$$


Trivially, if $a \in K$ then

$$
M(a)=|a| .
$$

Let us estimate $M(X-\alpha)$. By the previous study, in this case

$$
P_{k}(X)= \pm\left(X-\alpha^{2^{k}}\right)
$$

which shows that

$$
M(X-\alpha)=\max \{1,|\alpha|\} .
$$

Suppose now that

$$
P=(X-\alpha) Q(X)
$$

where $\alpha \in K$ (thus $Q(X) \in K[X]$ ). For any $K$ we have

$$
\mathcal{G}^{k}(P)=\mathcal{G}^{k}(X-\alpha) \cdot \mathcal{G}^{k}(Q)= \pm\left(X-\alpha^{2^{k}}\right) \cdot \mathcal{G}^{k}(Q) .
$$

Then Proposition 1 implies that

$$
|| \alpha^{2^{k}}|-1| L\left(\mathcal{G}^{k}(Q)\right) \leqslant(1+\operatorname{deg} Q) L\left(\mathcal{G}^{k}(Q)\right), \quad \text { for any } k \geqslant 1,
$$

thus if $|\alpha| \neq 1$ we get

$$
\max \{1,|\alpha|\} M(Q) \leqslant M(P),
$$

which, combined with (6) gives

$$
M((X-\alpha) Q(X))=M(X-\alpha) \cdot M(Q) .
$$

The remaining case, namely $|\alpha|=1$, can be treated using Lemma 3 below and leads to the the same result. This leads to the following proposition.

\section{Proposition 5 If}

$$
P=a\left(X-\alpha_{1}\right) \cdots\left(X-\alpha_{k}\right) R(X) \in K[X]
$$

where $\alpha_{1}, \cdots, \alpha_{k}$ belong to $K$, then

$$
M(P)=|a| \cdot \prod_{j=1}^{k} \max \left\{1,\left|\alpha_{j}\right|\right\} \cdot M(R) .
$$

In particular, if

$$
P=a\left(X-\alpha_{1}\right) \cdots\left(X-\alpha_{d}\right)
$$

splits completely in $K$ then we recover Mahler's definition

$$
M(P)=|a| \prod_{j=1}^{d} \max \left\{1,\left|\alpha_{j}\right|\right\} .
$$

Question 3 Is it always true that $M(Q \cdot R)=M(Q) \cdot M(R)$ ?

This question is also very important because it will help us to use the principle of "divide and conquer"!

Recall that we have

$$
M(P) \leqslant L(P), \quad M(P) \leqslant L\left(P_{1}\right)^{1 / 2}, \quad M(P) \leqslant L\left(P_{2}\right)^{1 / 4}, \ldots
$$

Notice also that when $P$ splits then

$$
L(P) \leqslant 2^{d} M(P) .
$$

Remark We could also have defined the measure by the similar formula

$$
M_{\infty}(P)=\lim _{k \rightarrow \infty}\left\|P_{k}\right\|_{\infty}^{2^{-k}},
$$

where, as above, $P_{k}=\mathcal{G}^{k}(P)$. It is clear that the sequence $\left(\left\|P_{k}\right\|_{\infty}^{-k}\right)$ is non-increasing, so that the limit exist, and we have

$$
M_{\infty}(P) \leqslant M(P)
$$


since $N\left(P_{k}\right) \leqslant L\left(P_{k}\right)$ for each $k$. When $K$ contains at least $d+1$ elements of absolute value equal to 1 , Proposition 3 shows that

$$
M_{\infty}(P)=M(P)
$$

and, in this case we have

$$
M(P) \leqslant N(P), \quad M(P) \leqslant N\left(P_{1}\right)^{1 / 2}, \quad M(P) \leqslant N\left(P_{2}\right)^{1 / 4}, \ldots .
$$

\section{Bounds for the Factors of Polynomials}

\subsection{The Case of a Linear Factor}

We first consider the case

$$
P(X)=(X-\alpha) Q(X),
$$

and follow Glesser (1990, pp. 71-75). Put

$$
P=\sum_{i=0}^{p} b_{j} X^{j} \quad \text { and } \quad Q=\sum_{i=0}^{p-1} a_{j} X^{j} .
$$

Then $b_{j}=a_{j-1}-\alpha a_{j}, 0 \leqslant j \leqslant p$, where $a_{p}=a_{-1}=0$.

This implies

$$
\left|a_{j}\right| \leqslant\left|b_{0}\right||\alpha|^{-j-1}+\left|b_{1}\right||\alpha|^{-j}+\cdots+\left|b_{j}\right||\alpha|^{-1}
$$

and

$$
\left|a_{j}\right| \leqslant\left|b_{p}\right||\alpha|^{p-j-1}+\left|b_{p-1}\right||\alpha|^{p-j-2}+\cdots+\left|b_{j+1}\right| .
$$

In the case $|\alpha| \geqslant 1$, using (9), we get

$$
|\alpha|\left|a_{k}\right| \leqslant \sum_{j=0}^{k}\left|b_{j}\right|
$$

and using (10), we get

$$
\frac{\left|a_{k}\right|}{|\alpha|^{p-k-1}} \leqslant \sum_{j=k+1}^{p}\left|b_{j}\right| .
$$

Combining these two results we get the following result.

Lemma 3 If $P(X)=(X-\alpha) Q(X)$ and $Q=\sum_{i=0}^{p-1} a_{j} X^{j}$ then, if $|\alpha| \geqslant 1$, we have

$$
\left(|\alpha|+\frac{1}{|\alpha|^{p-k-1}}\right)\left|a_{k}\right| \leqslant L(P)
$$

for $k=0,1, \ldots, p-1$.

Theorem 2 If $P(X)=(X-\alpha) Q(X)$, then

$$
H(Q) \leqslant \frac{\max \left\{1,|\alpha|^{p-1}\right\}}{1+|\alpha|^{p}} L(P), \quad \text { where } p=\operatorname{deg}(P) .
$$

Proof. If $|\alpha| \geqslant 1$, this is an easy consequence of Lemma 3 above. Just notice this lemma implies

$$
\left|a_{k}\right| \leqslant \frac{|\alpha|^{p-k-1}}{1+|\alpha|^{p-k}} L(P)
$$

and that

$$
\frac{|\alpha|^{t-1}}{1+|\alpha|^{t}} \leqslant \frac{|\alpha|^{t}}{1+|\alpha|^{t+1}}
$$

for all $t \geqslant 1$ when $|\alpha| \geqslant 1$. 
If $|\alpha|<1$, apply Lemma 3 to the polynomials $P^{*}$ and $Q^{*}$. Then

$$
P^{*}=(X-1 / \alpha)\left(-\alpha Q^{*}\right)
$$

and we get

$$
H\left(\alpha Q^{*}\right)=|\alpha| H\left(Q^{*}\right) \leqslant \frac{|\alpha|^{-(p-1)}}{1+|\alpha|^{-p}} L\left(P^{*}\right)=\frac{1}{|\alpha|^{p-1}+|\alpha|^{-1}} L(P),
$$

and the result follows.

Remark As notice by Ph. Glesser, Theorem 2 is sharp: if

$$
Q(X)=X^{p-1}+\alpha X^{p-2}+\cdots+\alpha^{p-2} X+\alpha^{p-1}
$$

then

$$
P(X)=(X-\alpha) Q(X)=X^{p}-\alpha^{p}
$$

and

$$
H(Q)=\max \left\{1,|\alpha|^{p-1}\right\}, \quad \text { and } \quad L(P)=1+|\alpha|^{p},
$$

so that equality holds in Theorem 2 for these two polynomials.

Corollary 3 If $P(X)=(X-\alpha) Q(X)$, then

$$
H(Q) \leqslant L(P)
$$

Remark With the notation of Theorem 2, in the case of polynomials with complex coefficients, Durand (1980) proved that

$$
N(Q) \leqslant \frac{p}{1+|\alpha|} N(P) .
$$

Notice also that Proposition 1 above provides a variant of Theorem 2.

\subsection{The Case of a Split Factor}

By the previous study we have:

Theorem 3 If $P$ is a monic polynomial and if $P=Q R$ where $Q$ and $R$ are monic, and $Q$ is completely split over $K$, then

$$
L(Q) \leqslant 2^{q} M(Q) \leqslant 2^{q} M(P) \leqslant 2^{q} L(P), \quad \text { where } q=\operatorname{deg}(Q) .
$$

\section{References}

Cauchy, A. L. (1891). Exercices de mathématiques, Quatrième année, De Bure Frères, Paris, 1829; reprinted in Oeuvres, Ser. II, Vol. IX, Gauthier-Villars, Paris.

Cerlienco, L., Mignotte, M., \& Piras, F. (1987). Computing the measure of a polynomial. J. Symb. Comp., 4(1), 21-34. http://dx.doi.org/10.1016/S0747-7171(87)80050-0

Davenport, J., \& Mignotte, M. (1990). On finding the largest root of a polynomial. M. A. N., 24(6), 693-696.

Diouf, I. (2007). Thèse de doctorat de mathématiques, Méthode de Dandelin-Graeffe et méthode de Baker. France: Strasbourg.

Diouf, I., Diakhate, B., \& Watt, A. O. (2013). Perturbation on Polynomials. Journal of Mathematics Research, 5(3), 51-55. http://dx.doi.org/10.5539/jmr.v5n3p51

Durand, A. (1980). A propos d'un théorème de S. Bernstein sur la dérivée d'un polynôme. C. Rend. Acad. Sc. Paris, Serie I, Math., 290, 523-525.

Glesser, Ph. (1990). Bornes pour les algorithmes de factorisation des polynômes, Thèse de Doctorat de mathématiques. France: Strasbourg.

Landau, E. (1905). Sur quelques théorèmes de M. Pétrovic̀ relatifs aux zéros des fonctions analytiques. Bull. Soc. Math. France, 33, 251-261.

Mignotte, M. (1991). An inequality of the greatest roots of a polynomial. Elemente der math.

Specht, W. (1938). Zur Theorie der algebraischen Gleichungen. Jahresber. Deutsch. Math., 5, 142-145. 


\section{Copyrights}

Copyright for this article is retained by the author(s), with first publication rights granted to the journal.

This is an open-access article distributed under the terms and conditions of the Creative Commons Attribution license (http://creativecommons.org/licenses/by/3.0/). 